\title{
Robust Density-Based Clustering to \\ Identify Metastable Conformational States of Proteins - Supporting Information -
}

\author{
Florian Sittel and Gerhard Stock \\ Biomolecular Dynamics, Institute of Physics, \\ Albert Ludwigs University, 79104 Freiburg, Germany
}

(Dated: April 6, 2016)

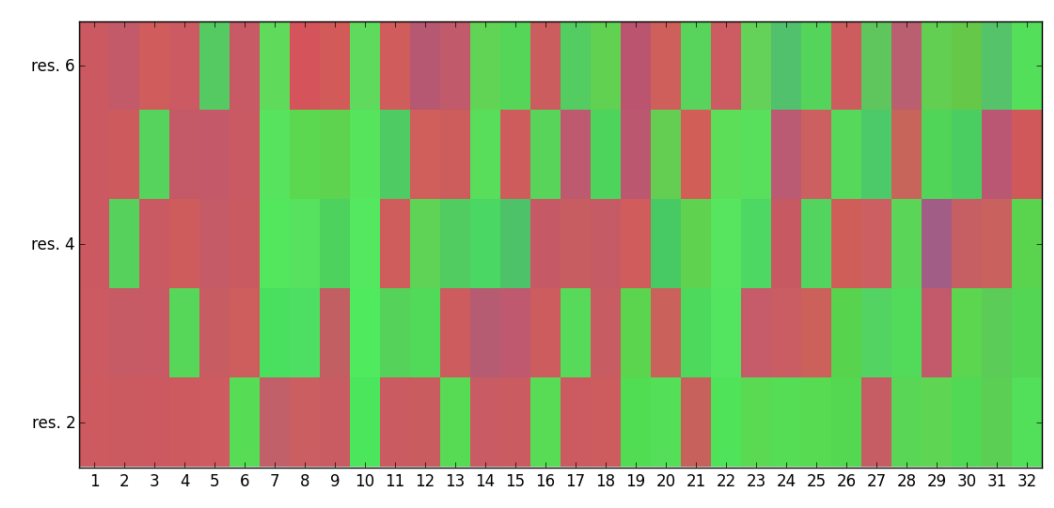

Figure S1: Ramacolor plot of $\mathrm{Ala}_{7}$, showing the dihedral angle content of its 32 metastable states. Red residues indicate $\beta$-sheet, green residues indicate $\alpha$-helical conformations.

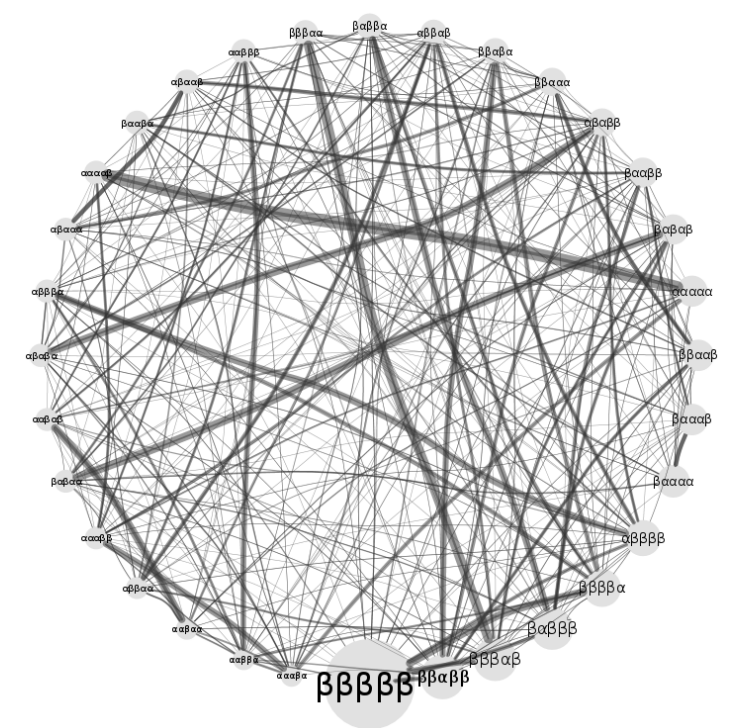

Figure S2: Transition network of Ala 7 . States are ordered counter-clockwise by population. Edge-thickness scales by transition rates, node sizes correspond to state populations. Node labels denote the dihedral conformation of the state, with residues either in $\beta$ - or $\alpha$-conformation. The network shows clearly that Ala 7 dynamics are essentially uncorrelated. 

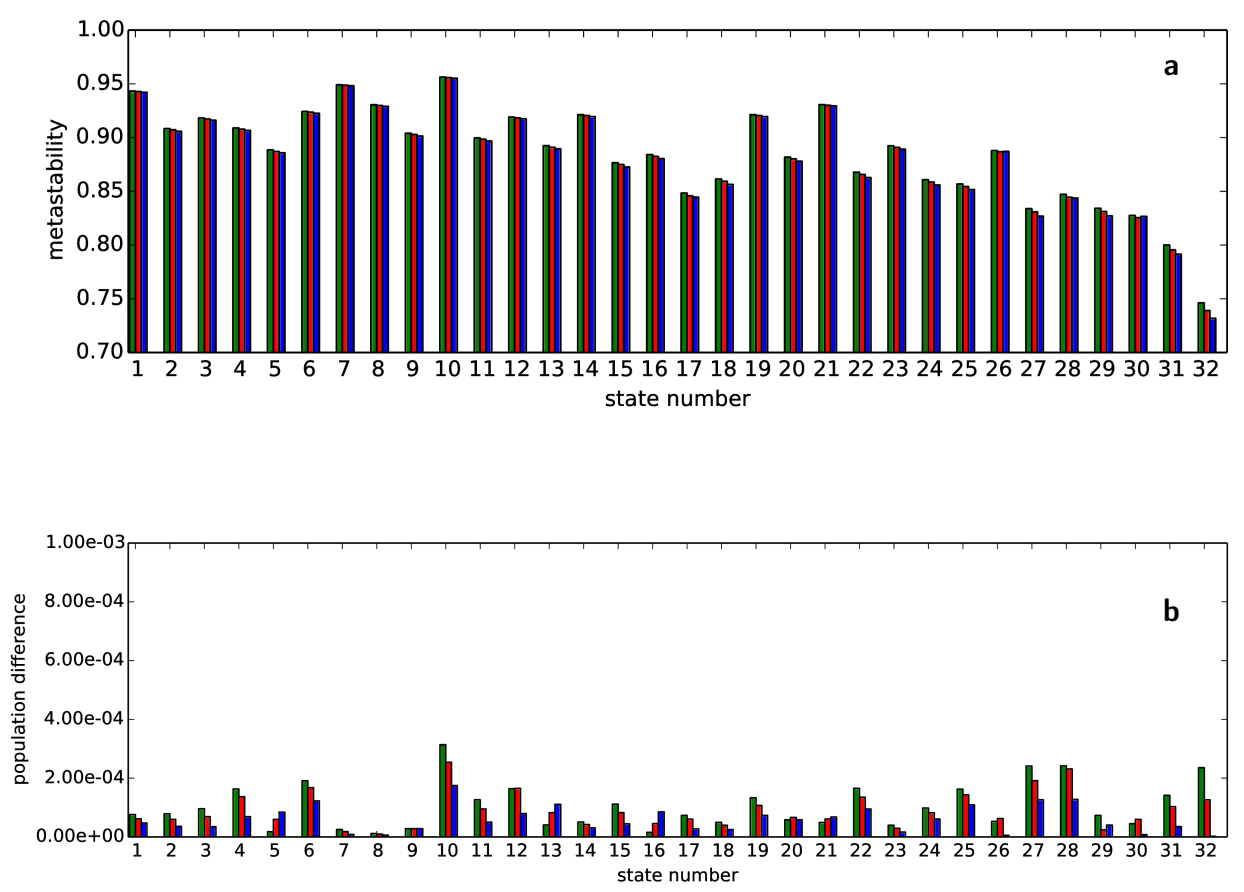

Figure S3: Verification of Chapman-Kolmogorov equation for Ala. Top diagram (a) shows metastabilities $\mathrm{T}_{i i}^{20} \mathrm{ps} / \tau(\tau)$ after propagation to $20 \mathrm{ps}$ for $\tau=1 \mathrm{ps}$ (green), $\tau=5 \mathrm{ps}$ (red) and $\tau=10 \mathrm{ps}$ (blue). Bottom diagram (b) shows differences in population after propagation of an initially equally distributed population $\mathbf{p}$. Compared are the same lag times as in (a) to a propagation with $\mathrm{T}(\tau=20 \mathrm{ps})$, i.e., pop. diff. $\equiv\left\|\mathbf{p T}^{20 \mathrm{ps} / \tau}(\tau)-\mathbf{p T}(\tau=20 \mathrm{ps})\right\|$.
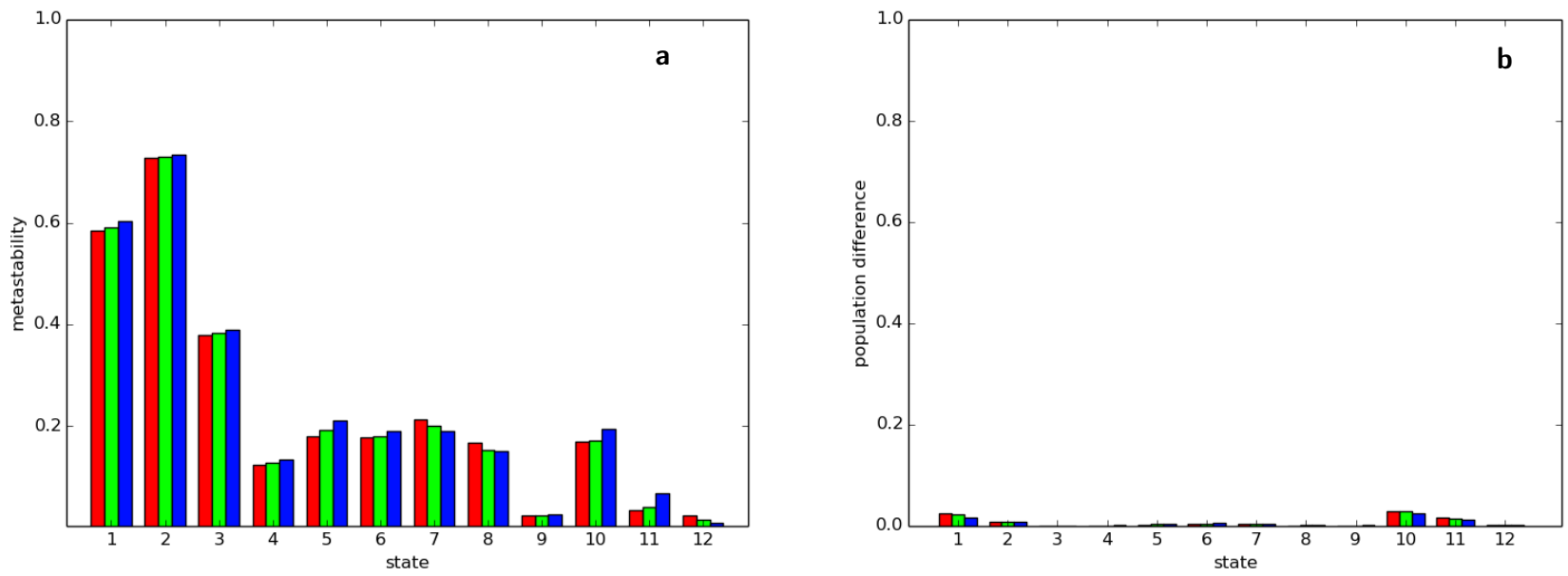

Figure S4: Verification of Chapman-Kolmogorov equation for HP-35. a: Metastabilities $\mathrm{T}_{i i}^{160 \mathrm{~ns} / \tau}(\tau)$ after propagation to $160 \mathrm{~ns}$ for $\tau=10 \mathrm{~ns}$ (green), $\tau=20 \mathrm{~ns}$ (red) and $\tau=40 \mathrm{~ns}$ (blue). b: Differences (relative) in population after propagation of an initially equally distributed population p. Compared are the same lag times as in (a) to a propagation with $\mathrm{T}(\tau=160 \mathrm{~ns})$, i.e., pop. diff. $\equiv\left\|\mathbf{p} \mathrm{T}^{160 \mathrm{~ns} / \tau}(\tau)-\mathbf{p T}(\tau=160 \mathrm{~ns})\right\|$ 


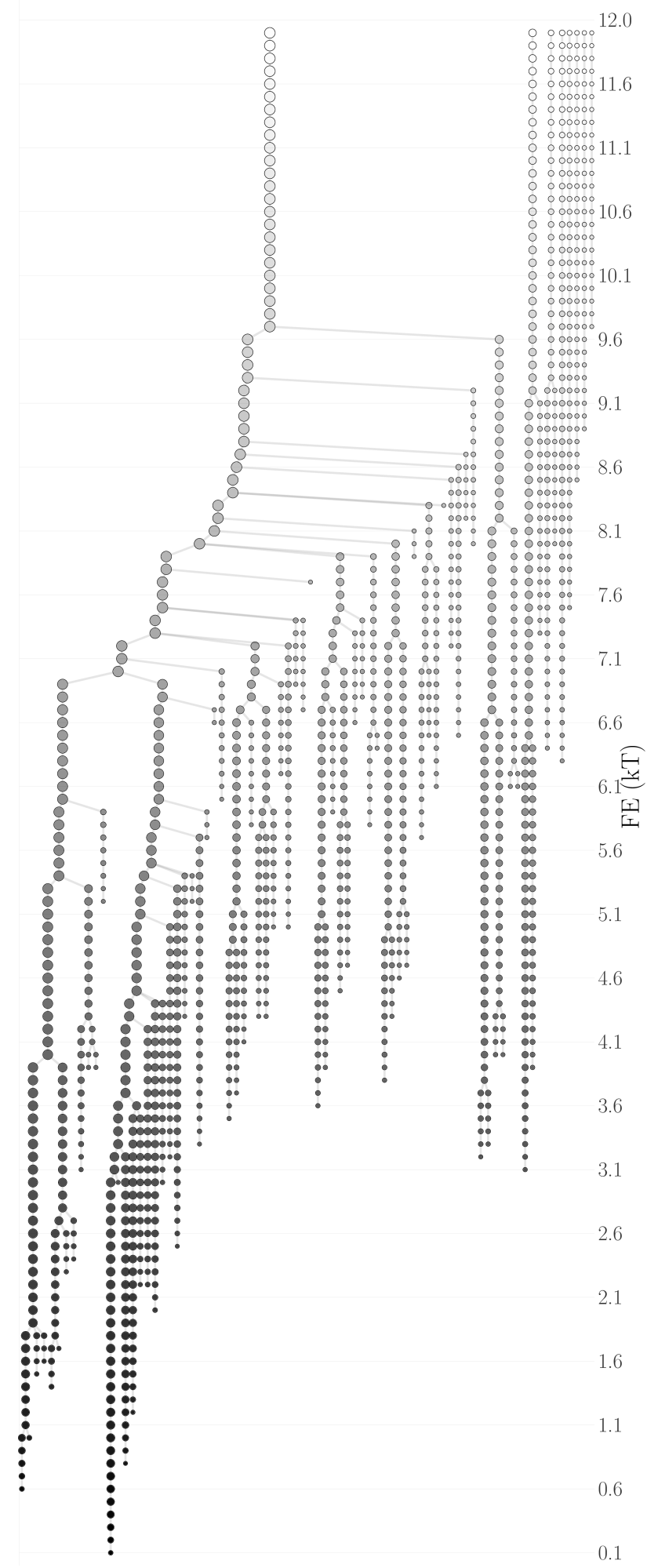

Figure S5: Density network for BPTI with free energy levels from $0.1 \mathrm{kT}$ to $12.0 \mathrm{kT}$ in steps of $0.1 \mathrm{kT}$. 


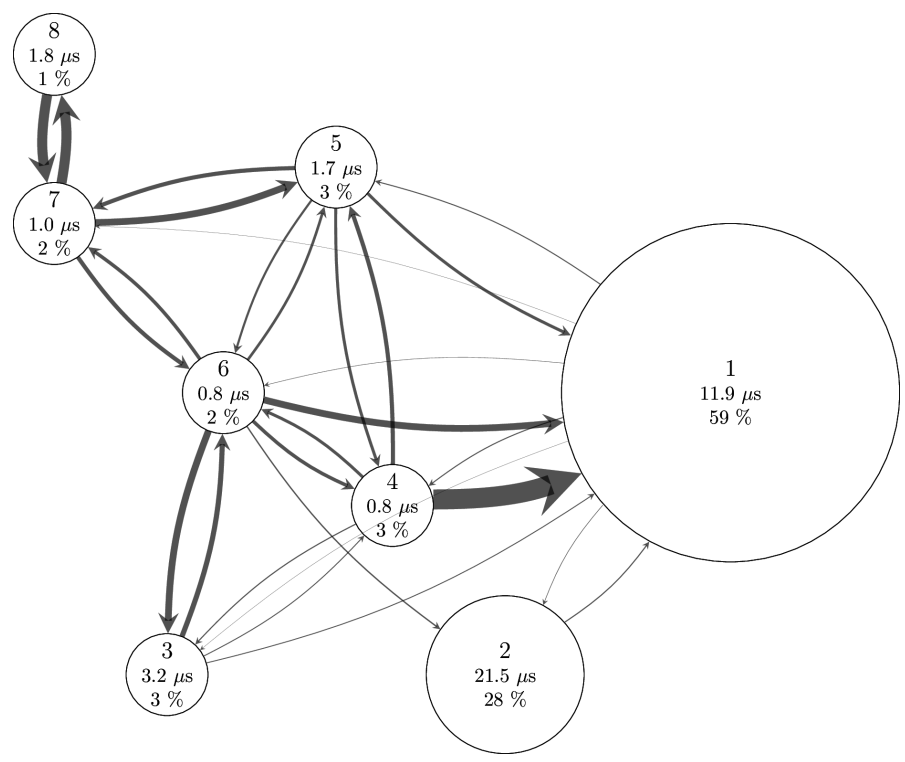

Figure S6: Markov state model of BPTI resulting from density-based geometric clustering and successive dynamical clustering via MPP on reduced dihedral-angle PCs.

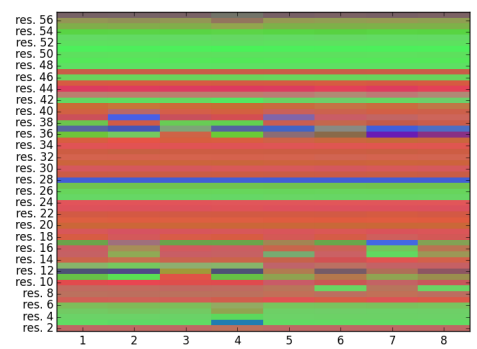

a

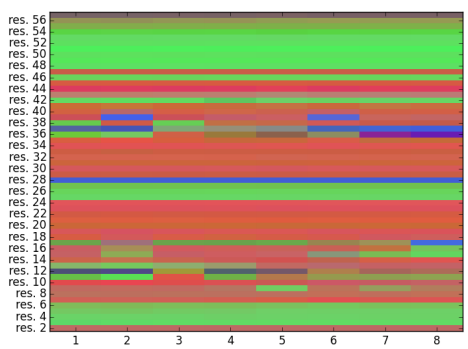

b

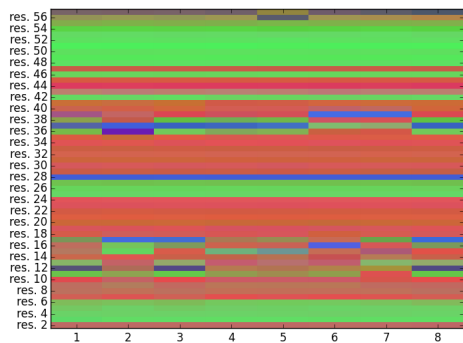

C

Figure S7: Dihedral angle content for BPTI after: primitive clustering (a), density-based geometric microstate generation and successive dynamical clustering via MPP (b) and density-based geometric clustering without dynamical corrections $(\mathbf{c})$. 